\title{
Innovar las formas de enseñanza + Involucrar a los alumnos = Provocar aprendizaje significativo
}

\author{
Innovate teaching ways + Involve students $=$ Provoke meaningful learning
}

Ana Y. Morales-Flores ${ }^{a}$, Laura E. Pérez-Ramírez, ${ }^{b}$

\begin{abstract}
:
Nowadays, Information and Communication Technologies (ICT) are a didactic resource and a communication channel where language teachers can extract a groundbreaking utility, as it allows both the teacher and the student to come up with their creativity during the teaching-learning process. On the other hand, more practical and more suitable didactic materials that result in a more personalized teaching practice, that allows to provide feedback on the progress of each student, emerge to reach more students in less time, and also to introduce a variety of motivational learning elements.

In addition, another interesting element about technology is its feasibility to be used in the classroom, as it offers students the possibility to extend their learning opportunities beyond the classroom in a way in which it suits their personal space, thanks to the large amount and universal access to learning resources that are available through internet sites which are also useful and free.
\end{abstract}

Throughout the innovation process of our class environment, we found several apps that can be used to promote a positive impact on students and the results were not just meaningful but also fascinating.

\section{Keywords:}

Apps, creativity, ICT, innovating, meaningful

\section{Resumen:}

Hoy en día las Tecnologías de la Información y la comunicación (TIC) son un recurso didáctico y una vía de comunicación de la cual los docentes de lenguas podemos extraer una utilidad sin precedentes. Todo esto permite aplicar creatividad tanto del docente como del alumno en el proceso de enseñanza-aprendizaje; ya que las TIC nos proporcionan material práctico y didáctico cuyo resultado es una enseñanza más personalizada, aportando retroalimentación del progreso de cada alumno, a la vez que permite alcanzar a más alumnos en menos tiempo, así como también introducir una variedad de elementos de motivación en el aprendizaje.

Otro de los elementos interesantes sobre la tecnología es que además de ser factible de utilizar en el aula presencial, ofrece la posibilidad de que los alumnos extiendan su aprendizaje más allá del aula, esto es, a su espacio personal, gracias a la gran cantidad y al acceso universal a recursos de aprendizaje a través de sitios de internet que son además de útiles, gratuitos.

Durante el proceso de innovación de nuestro entorno de clase, pudimos encontrar varias aplicaciones que se pueden utilizar para promover un impacto positivo en los alumnos y los resultados no solo fueron significativos sino también fascinantes.

\section{Palabras clave}

Apps, creatividad, innovar, significativo, TIC

\section{INTRODUCCION}

En la Escuela Superior Huejutla existe un bajo interés por parte de los alumnos para el aprendizaje de inglés como lengua extranjera. Es necesario encontrar medios y recursos que motiven a los estudiantes en el aprendizaje del idioma inglés, y que efectivamente se convierta en un aprendizaje significativo.

Cabe destacar, que la gran mayoría de estudiantes proviene de zonas indígenas; además, algunos no habían tomado una clase

a Corresponding author, Universidad Autónoma del Estado de Hidalgo, México, https://orcid.org/0000-0001-9749-8965, Email: ana_morales9717@uaeh.edu.mx

${ }^{b}$ Universidad Autónoma del Estado de Hidalgo, México, https://orcid.org/0000-0002-1593-2543, Email: 1perezr@uaeh.edu.mx 
de inglés hasta que llegaron a la universidad, su lengua materna es el náhuatl, su segunda lengua es el español y están sujetos a aprender una tercera lengua que es el inglés.

Por lo que en el presente trabajo se sugieren algunas apps, plataformas virtuales, y se explica la aplicación de las mismas involucrando a los alumnos, provocando así el aprendizaje significativo en ellos.

Eran de esperarse resultados positivos, sin embargo, fueron más allá de los esperados académicamente. Los alumnos descubrieron que los celulares pueden tener un nuevo uso, con propósitos educativos, pusieron en práctica la competencia del trabajo colaborativo y el valor de la solidaridad, al poder ayudar a los compañeros de clases que presentaban dificultades para instalar una nueva App.

\section{ROL DEL DOCENTE}

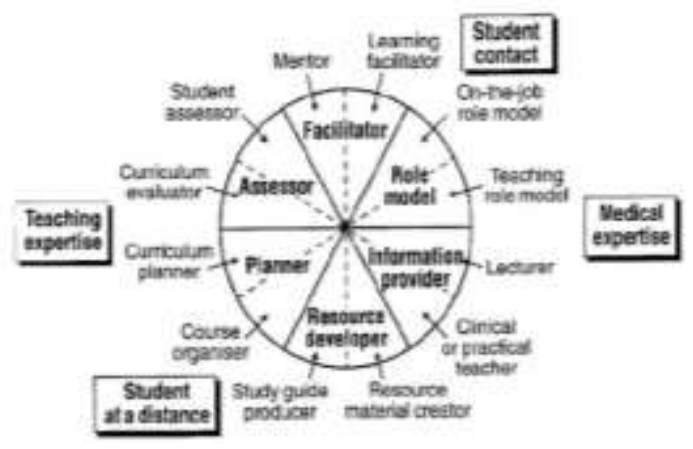

Imagen 1. Rol del docente.

Tomada de https://www.researchgate.net/figure/The-12-th-1roles-of-the-student-as-proposed-by-Ioannina-3rd-yearmedical-students_fig3_221752749

Para los nativos digitales el uso de la tecnología es algo natural en todos los aspectos de sus vidas. Aretio (2007) señala que "el uso indiscriminado de tecnologías aplicadas a la educación, porque están de moda, nunca será garantía de éxito, aunque sí un poderoso instrumento, que bien utilizado por expertos tecnólogos de la educación, puede producir excelentes resultados sobre la base de un determinado modelo pedagógico."

En este sentido, no debemos olvidar que el papel del profesor en la enseñanza de idiomas es esencial. El profesor es quien crea las condiciones de aprendizaje que son adecuadas a los objetivos del alumno, sirve de guía, facilitador y apoyo. De igual manera, el docente es el elemento que propicia un clima positivo en un grupo de alumnos para el intercambio personal, promoviendo el desarrollo de las habilidades comunicativas y la creatividad en los alumnos.

Este enfoque innovador en el cual los docentes se encuentran en medio del proceso de cambio hacia el uso de la tecnología en las aulas, comprende inherentemente cambios significativos en el aspecto metodológico de su labor. El resultado que buscamos en la Escuela Superior Huejutla es una mejora en el proceso de aprendizaje de los estudiantes y que este aprendizaje resulte ser significativo.

\section{LOS RECURSOS TECNOLÓGICOS, ;TODOS, ¿CUALQUIERA, O CUÁLES?}

Aún existe entre los docentes una cierta reticencia a emplear de manera cotidiana los recursos tecnológicos digitales, en parte debido a la incertidumbre que causa el desconocimiento o el estar poco familiarizados con ellos. Las preguntas surgen inevitablemente, ¿Tal vez me va a consumir más tiempo incorporar este recurso en mi planeación? ¿El resultado será tan bueno que valga la pena? Estas cuestiones, entre otras, cruzan la mente del docente ante la sugerencia del uso de medios digitales en su práctica diaria.

Tales cuestionamientos son válidos. Sin embargo, debemos recordar que nuestra labor como docentes es como un largo viaje que está hecho de variadas estaciones, de cambios de medio de transporte, tal vez hasta de algunas equivocaciones de ruta, para después hallar la correcta, pero si llevamos el equipaje apropiado, disfrutaremos del viaje en sí mismo y arribaremos felizmente a un destino.

O tal vez no existen muchos recursos en la institución con la cual colaboramos.

Aún si este fuese el caso, la recomendación es aprender acerca de los recursos tecnológicos que podemos incorporar a nuestras clases. De esa manera, llegaremos a tener un acercamiento a ellos, hasta familiarizarnos con su uso.

Otra recomendación que tomamos en cuenta en nuestro caso fue utilizar lo que está disponible. Seguramente conocen el concepto de B-Y-O-D, bring your own device. Usando ese principio, fue posible diseñar actividades centradas en ese concepto, utilizando las posibilidades tecnológicas de los propios estudiantes.

\section{INNOVAR LAS FORMAS DE APRENDIZAJJE}

En la Escuela Superior Huejutla se tomaron en consideración algunos puntos al momento de elegir e incorporar tecnología en nuestras clases.

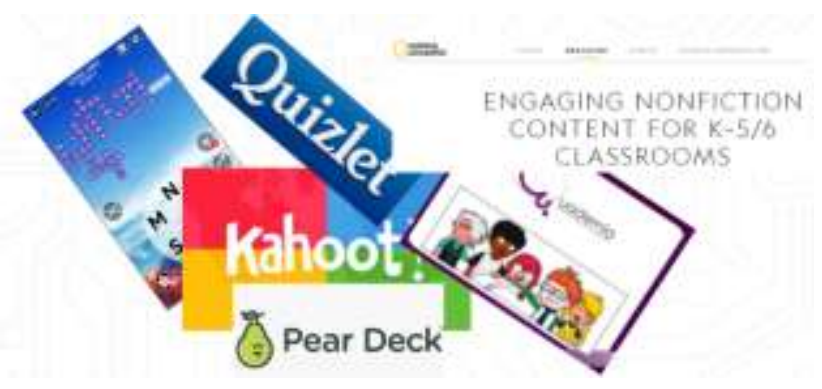

Imagen 2. Apps facilitadoras del aprendizaje del inglés Tomadas de:

https://play.google.com/store/apps/details?id=in.myinnos.AppM anager\&hl=es_MX

$>$ Primero, la disponibilidad de recursos en la institución. El no contar con una amplia gama de recursos tecnológicos en la institución fue un aliciente 
para explorar los recursos a nuestro alcance fuera de ella. En este punto también se consideró el concepto BYOD (bring your own device), para diseñar actividades centradas en ese concepto, utilizando las posibilidades tecnológicas de los estudiantes.

$>$ En segundo lugar, el uso de los recursos tecnológicos no puede ser de ninguna manera indiscriminado. Es necesario el buen criterio del docente, para que se obtenga de tales recursos el máximo beneficio para los estudiantes.

$>$ En tercer lugar, la tecnología debe estar alineada con el curriculum, llámese programa de la asignatura, syllabus, contenido, etc. Debe haber un propósito claro en el uso de tecnología. Algunas de las preguntas detonantes fueron, ¿Qué vocabulario deben conocer? ¿En qué situaciones deben saber emplear el idioma? ¿Qué tipo de actividades queremos que desarrollen en las cuatro habilidades básicas: leer, escuchar, escribir y hablar?

Después de tales consideraciones buscamos la tecnología adecuada, que sirviese de apoyo para los objetivos de enseñanza y aprendizaje.

De esta manera buscamos poner en práctica de manera eficaz la fórmula:

\section{Innovar las formas de enseñanza + Involucrar a los alumnos $=$ Provocar un aprendizaje significativo}

El Dr. Rubén Puentedura creó el modelo SAMR, Substitution, Augmentation, Modification And Redefinition, el cual puede ayudar al docente a seleccionar las herramientas tecnológicas adecuadas para el propósito específico para el cual serán utilizadas.

La siguiente figura muestra de manera esquemática el modelo SAMR.

\section{THE SAMR MODEL} Or. Pushen R. Pusmodum
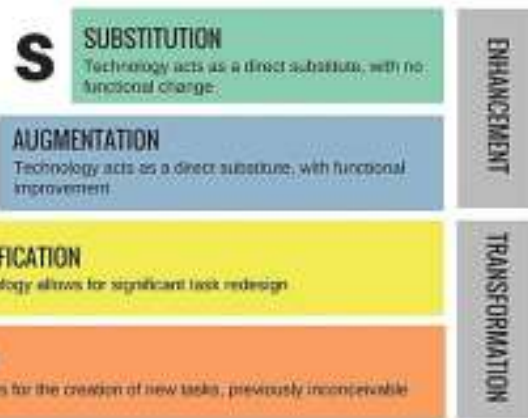

$\mathbf{R}$

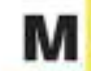

MODIFCATION

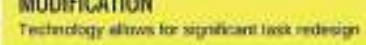

\section{AEDEFINITIN}

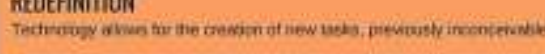

Imagen 3: Modelo SAMR

Imagen tomada de

https://en.wikiversity.org/wiki/Instructional_design/SAMR_Mo del/What_is_the_SAMR_Model\%3F

El propósito de este modelo es ayudar a los docentes a determinar el nivel de impacto o integración de tecnología en el proceso de enseñanza y aprendizaje. Una vez que se ha decidido el propósito para el cual se utilizará el recurso tecnológico, determinamos si éste ha de resaltar (por medio de Sustituir o Aumentar), o transformar el proceso de enseñanza y aprendizaje (a través de Modificar y/o Redefinir).

Se consideró la siguiente clasificación como apoyo en la identificación y clasificación de las diferentes herramientas a manera de tener una visión más clara, más amplia, de todas ellas, y de ese modo, poder decidir cuál herramienta incorporar en clase.

Esta tabla es solamente ilustrativa, no se pretende de ninguna manera afirmar que incluye todas las posibles herramientas que existen y que están al alcance de los docentes.

\begin{tabular}{|c|c|}
\hline CATEGORIA & HERRAMIENTAS \\
\hline $\begin{array}{l}\text { Aplicaciones de } \\
\text { procesamiento de texto. }\end{array}$ & $\begin{array}{l}\text { Google docs } \\
\text { Microsoft Word } \\
\text { Word Cloud Generators }\end{array}$ \\
\hline $\begin{array}{l}\text { Software de organización } \\
\text { y generación de ideas. }\end{array}$ & $\begin{array}{l}\text { Webspiration } \\
\text { Inspiration } \\
\text { SMART tools }\end{array}$ \\
\hline $\begin{array}{l}\text { Recolección y análisis de } \\
\text { datos. }\end{array}$ & $\begin{array}{l}\text { Google forms } \\
\text { Survey Monkey } \\
\text { Poll everywhere }\end{array}$ \\
\hline $\begin{array}{l}\text { Software de comunicación } \\
\text { y colaboración. }\end{array}$ & $\begin{array}{l}\text { Skype } \\
\text { Facetime } \\
\text { Typewith.me } \\
\text { Facebook } \\
\text { Twitter } \\
\text { LMS } \\
\text { Cuadernia }\end{array}$ \\
\hline Medios de instrucción. & $\begin{array}{l}\text { Brain POP } \\
\text { Discovery Education } \\
\text { Streaming } \\
\text { Khan Academy } \\
\text { TEDx Talks } \\
\text { TED talks } \\
\text { VOA }\end{array}$ \\
\hline $\begin{array}{l}\text { Herramientas de creación } \\
\text { de multimedios. }\end{array}$ & $\begin{array}{l}\text { PowerPoint } \\
\text { Prezi } \\
\text { Keynote } \\
\text { VoiceThread } \\
\text { iMovie } \\
\text { blogs } \\
\text { vlogs }\end{array}$ \\
\hline
\end{tabular}




\begin{tabular}{|c|c|}
\hline & $\begin{array}{l}\text { Pow Toon } \\
\text { Animaker } \\
\text { Synfig Studio } \\
\text { Scratch }\end{array}$ \\
\hline $\begin{array}{l}\text { Medios instruccionales } \\
\text { interactivos. }\end{array}$ & $\begin{array}{l}\text { English Central } \\
\text { Duolingo } \\
\text { Busuu } \\
\text { ImmerseMe } \\
\text { Quizizz } \\
\text { Quizlet } \\
\text { Pear Deck } \\
\text { Hot Potatoes } \\
\text { Kahoot! } \\
\text { Google Classroom } \\
\text { Educaplay }\end{array}$ \\
\hline $\begin{array}{l}\text { Recursos de bases de datos } \\
\text { y referencia. }\end{array}$ & $\begin{array}{l}\text { Wikipedia } \\
\text { Wolfram/Alpha } \\
\text { Gapminder } \\
\text { Rubistar }\end{array}$ \\
\hline Tecnología cinestésica. & $\begin{array}{l}\text { Nintendo Wii } \\
\text { Xbox Kinect } \\
\text { GPS } \\
\text { Classcraft }\end{array}$ \\
\hline
\end{tabular}

Tabla 1: Clasificación de herramientas digitales. Creación propia de las autoras

A continuación, se muestran algunos ejemplos de las diferentes etapas del proceso de enseñanza y aprendizaje con uso de las herramientas tecnológicas.

\begin{tabular}{|l|l|}
\hline \multicolumn{1}{|c|}{ ETAPA } & \multicolumn{1}{|c|}{$\begin{array}{c}\text { HERRAMIENTA } \\
\text { TECNOLÓGICA }\end{array}$} \\
\hline $\begin{array}{l}\text { Estimulación del } \\
\text { conocimiento previo }\end{array}$ & Fotografías digitales, videos \\
\hline $\begin{array}{l}\text { Organización } \\
\text { administración } \\
\text { información }\end{array}$ & de Presentación en PowerPoint \\
\hline Entrega de instrucciones & Presentación en Word \\
\hline $\begin{array}{l}\text { Práctica y colaboración } \\
\text { Producción y demostración } \\
\text { de las habilidades }\end{array}$ & $\begin{array}{l}\text { Portafolio digital, blogs, wikis, } \\
\text { foros en Google classroom }\end{array}$ \\
\hline \begin{tabular}{l} 
Evaluación \\
\hline
\end{tabular} & y
\end{tabular}

retroalimentación

Tabla 2: Clasificación de herramientas a aplicar en clase. Creación propia de las autoras.

\section{INVOLUCRAR A LOS ALUMNOS}

La gran mayoría de la población estudiantil hoy en día utiliza un Smartphone, sin embargo, el uso de estos dispositivos muchas veces se limita a las redes sociales como WhatsApp, Facebook, Instagram, entre otras.

Por lo que se decidió involucrar otras aplicaciones en las que los alumnos participasen de manera individual o colaborativa y que el producto de largas horas de práctica tuviese impacto positivo en su aprendizaje.

Para que el alumno pueda comprender correctamente un proceso debe ser involucrado desde el inicio del curso.

Para lo que se consideran fundamentales los siguientes tres puntos:

1. Tomar ventaja de los recursos: en la Escuela Superior de Huejutla hoy en día se cuenta ya con internet de buena calidad y velocidad, así como computadoras y pantallas inteligentes, mismas que pueden ser conectadas a la red, lo que facilita la implementación de la tecnología en el aula.

2. 7 de cada 10 alumnos provienen de zonas marginadas: por increíble que parezca la gran mayoría jamás ha tomado una clase de inglés; sin embargo, cuentan con el náhuatl como lengua materna, una segunda lengua que es el español, y por consecuencia el inglés sería su tercera lengua, lo cual parece ser una ventaja que les facilita el proceso de aprendizaje. Debido a eso, en este punto se concientiza sobre la facilidad que se presupone que los estudiantes tendrán para adquirir una tercera lengua.

La adquisición de una tercera lengua L3 ha sido motivo de investigación en los últimos años. En este sentido los estudios reivindican que los procesos de ASL (L2) y ATL (L3) presentan diferencias, aunque con algunos puntos en común. (Jessner, 1999; Cenoz, 2003; Hufeisen y Marx, 2004).

\section{Cenoz (2003) señala que}

"...third language learners have more language experience at their disposal as second language learners are influenced by the general effects of bilingualism in cognition, and have access to two linguistic systems when acquiring a third language"

3. Al menos 9 de cada 10 alumnos cuenta con un Smartphone: por lo regular lo utilizan únicamente para redes sociales, por lo que enseñarles a utilizarlo con propósitos educativos es el nuevo reto y es en este punto en donde se les involucra para dar lugar al aprendizaje significativo.

Encaminar en el uso de herramientas digitales a las nuevas generaciones conlleva que los estudiantes aprendan a darle el 
uso adecuado a la nueva tecnología, generando así educandos autónomos, autodidactas y competentes para el mundo real que les espera al terminar la escuela.

Teniendo en cuenta los tres aspectos anteriores el rol como docente sugiere implementar las siguientes acciones para aplicar la tecnología:

> Analizar los objetivos de la clase, unidad o tema, es decir; saber en qué momento aplicar las plataformas o aplicaciones educativas.

> Ser empático con las circunstancias de los alumnos, por ejemplo, no todos saben cómo usar la app store de google y se les debe enseñar

$>$ Diseñar la sesión de acuerdo al nivel de inglés de los alumnos; no todos tienen el mismo nivel cuando ingresan a la educación superior, por lo tanto, es importante considerar que el impacto va a variar de acuerdo a sus estilos de aprendizaje.

$>$ Procurar la motivación diaria, ya que es este el rol principal del docente, considerando que es el principal exponente en el aula, del idioma a aprender, debemos tomar en cuenta que en este punto el plan realizado puede tener éxito o puede no funcionar si no lo hacemos de la forma correcta.

Como ejemplo de todas las consideraciones mencionadas en el apartado de "Involucrar a los alumnos" de este documento se retoma lo referente a la aplicación de apps y/o plataformas dentro de la clase de inglés describiendo a continuación específicamente la plataforma Kahoot!

\section{KAHOOT!}

Esta plataforma permite crear tests de diferentes tipos, opción múltiple, falso y verdadero o bien orden de palabras.

Las ventajas de poder personalizar los modelos de los tests y el tiempo de respuesta, contribuye en cierta medida a preparar a los estudiantes para trabajar contra el reloj; como parte de ciertas habilidades que deberán poner en práctica al realizar el examen TOEFL, pues en este examen además de preparar a los estudiantes para que evidencien sus habilidades en reading, listening $\mathrm{y}$ en use of the language, el factor tiempo es determinante por lo que los estudiantes deben estar preparados para responder de la manera correcta en el tiempo indicado.

Considerando ese factor, al término de cada lección se administraron exámenes, en donde se les indicaba que debían considerar trabajar contra el reloj, en intervalos de 10 segundos, 20 segundos y 30 segundos.

La primera experiencia fue emocionante para los alumnos, debido a que era algo completamente novedoso para ellos, sin embargo, en la segunda administración, habían empatizado con la aplicación y mostraban sentirse más seguros al momento de leer y responder antes de que el reloj marcara cero.

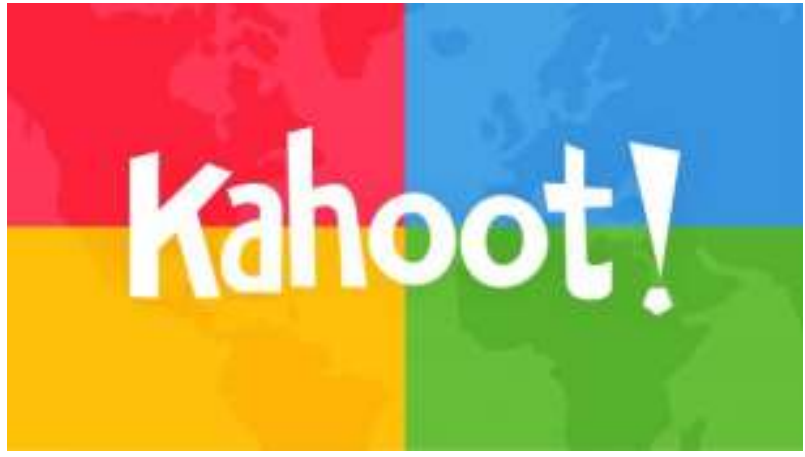

Imagen 4: Sitio web Kahoot! Aplicado en clase. Tomado de: https://kahoot.com/

Al realizar el ¡Kahoot day! en la clase de inglés, se les ha dado a nuestros alumnos un nuevo propósito de uso de sus celulares, y es que desde que se implementó esta plataforma como parte de sus clases para practicar, ha sido notable la participación entusiasta de los estudiantes.

Vocabulary contest day; se sugiere el uso de la aplicación llamada "wordscapes", la cual le permite al alumno formar palabras con cierto número de letras (tiene un límite cada nivel), la aplicación cuenta con un diccionario adicional en el que explica el significado de la palabra y sus diferentes usos (como verbo, adverbio, adjetivo, etc.) y está completamente en inglés.

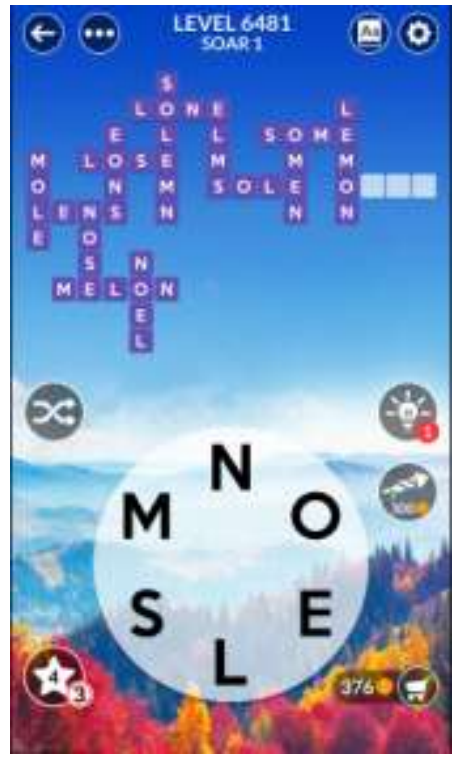

Imagen 5: App Wordscapes aplicada en clase

Tomada

https://play.google.com/store/apps/details?id=com.peoplefun.w ordcross \&hl=es

Al momento del inicio de una lección, es importante que la información se entregue a los alumnos de manera atractiva, que resulte que de algún modo se logre pregnancia en el estudiante para asegurar que permanecerá en la memoria del alumno por el tiempo suficiente para que aprenda su uso y una vez que ya ha sido utilizada la información de manera práctica, pasará a formar parte de la memoria a largo plazo, lo que favorece el aprendizaje significativo. 
Para este propósito hemos podido utilizar herramientas tales como Pear Deck. La imagen que se muestra es ilustrativa del vocabulario que se entrega al inicio de la lección a fin de familiarizar a los estudiantes con él mismo.

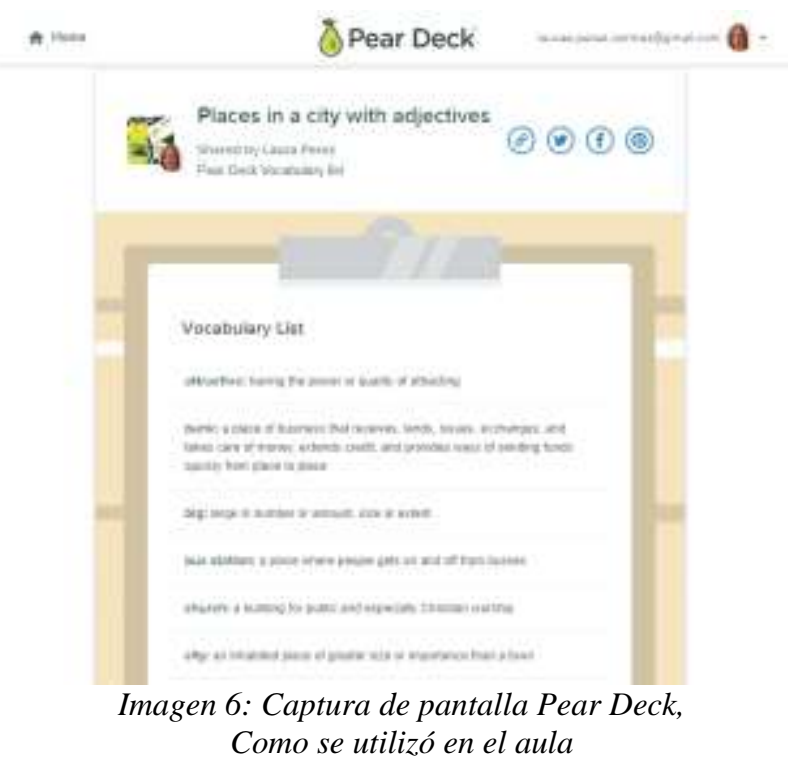

\section{PROVOCAR UN APRENDIZAJE SIGNIFICATIVO}

Una vez que el alumno comprende lo que está haciendo se genera una serie de emociones significativas, lo que lo lleva a querer explorar y descubrir aún más cosas. Esto se afirma con base en las premisas de:

$>$ Enjoy: El hecho de aplicar algo nuevo y diferente en una clase ordinaria de inglés hace que los estudiantes disfruten de la novedad y se sienten parte de una experiencia diferente.

> Relax: Los estudiantes disfrutan cuando salen de la rutina, cuando las clases se hacen más amenas y en consecuencia hay un bajo nivel de estrés, es entonces que se activa su receptividad al aprendizaje y que ellos se dan cuenta de que son capaces de aprender lo que siempre creyeron que era muy complicado o imposible, generando a la vez proactividad.

$>$ Solve: Resolver incógnitas mediante nuevos descubrimientos empodera a los estudiantes; por lo tanto, se sienten felices y motivados a diseñar sus propios retos.

\section{RESULTADOS DESEABLES EN LA ESHU}

En resumen, los alumnos tuvieron la oportunidad de reflexionar sobre el nuevo propósito del uso de sus celulares. Por ejemplo, al poco tiempo de que se implementó el uso de Kahoot y wordscapes, los alumnos por si mismos iniciaron la gestión de su propio aprendizaje, buscando más ejemplos y recolectando palabras, incrementando así su vocabulario.

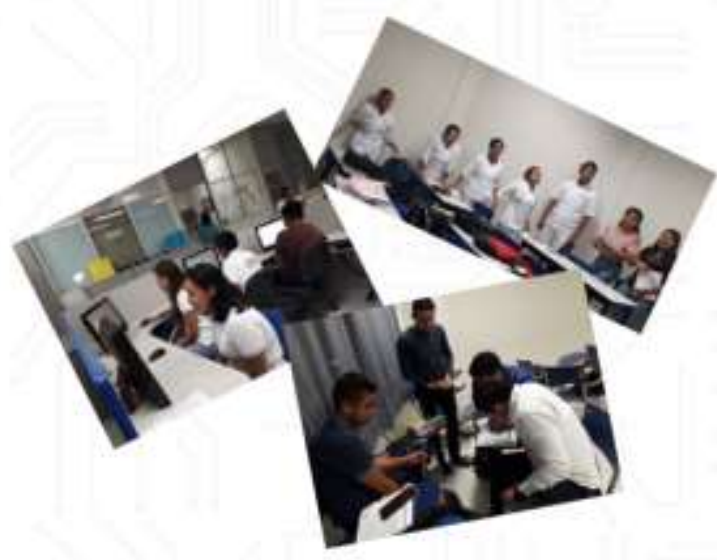

Imagen 6: Alumnos de la Escuela Superior de Huejutla

$>$ Fue posible evidenciar como los estudiantes se enamoraban del idioma inglés tan pronto lo iban comprendiendo, después de que finalmente se familiarizaron con los nuevos procedimientos de inclusión de apps y sitios web, los estudiantes mostraron más empatía y el ambiente áulico se tornó más positivo en comparación con los primeros días de clases.

$>$ Trabajar contra el reloj fue fundamental para preparar a los estudiantes para un examen de certificación. En la Escuela Superior de Huejutla, se habían implementado previamente diversas estrategias para que el alumno estuviera preparado para una prueba TOEFL, sin embargo, el tiempo seguía siendo un factor determinante para que los estudiantes no lograran concluir la prueba completa.

El que los alumnos se acostumbraran a trabajar contra el tiempo, les ayudó a manejar las secciones de la prueba TOEFL, debido a que administraban de mejor manera el tiempo para realizar los ejercicios.

$>$ Los estudiantes ya no temen a nuevos retos; el saber cómo utilizar aplicaciones, desde descargarlas, hasta instalarlas, etc., les dio la autonomía suficiente para investigar más y estar listos para cualquier reto que se les pudiera presentar en el futuro.

$>$ Los alumnos se volvieron conscientes de que es posible hacer uso del celular con fines educativos, pues saben que lo pueden utilizar para aprender la materia que consideraban difícil $y$ ha sido determinante para disminuir la pérdida de tiempo en las redes sociales.

\section{EVALUAR LOS RECURSOS}

En la Escuela Superior Huejutla, la aplicación de estrategias empleando medios y herramientas tecnológicas y digitales no es el final del camino. Aún está pendiente evaluar el impacto a largo plazo de estas estrategias ya que lo que aquí se reporta fue documentado durante un año,(es decir dos semestres). Por lo que los resultados no son concluyentes, sin embargo, sí son muy alentadores. 


\section{REFERENCIAS}

[1] American Psychological Association. (2012). Publication Manual of the American Psychological Association. Washington, DC: American Psychological Association.

[2] Aretio, L., Corbella, M. \& Figaredo, D. (2007). De la educación a distancia a la educación virtual.

[3] Carretero Ramos, A "Las TICs en el aula de inglés".

[4] Cebrián, Manuel "Nuevas competencias para la formación inicial y permanente del profesorado."

[5] Cenoz, J. (2003). "The additive effect of bilingualism on third language acquisition: A review". International Journal of Bilingualism. 7, 71-87.

[6] Hufeisen, B. y Marx, R. (2004). "A critical overview of research on third language acquisition and multilingualism published in the German language". International Journal of Bilingualism, 1:2, 141-154.

[7] Jessner, U. (1999), "Metalinguistic awareness in multilinguals: cognitive aspects of third language learning". Language awareness, 8:3, 201-209

[8] Revista Iberoamericana de la Educación. $N^{\circ}$ 44, 2007, consultada en file:///C:/Users/sku-60393/Downloads/2312Texto\%20del\%20artículo-975-1-10-20180209.pdf

[9] Sánchez García, L (2.004) "Las TICs y la formación del profesorado en la Educación Secundaria". 